\section{Contrast of resource frontiers}

\author{
G. de Q. Robin
}

\author{
Arctic and Antarctic: A Modern \\ Geographical Synthesis. \\ By David Sugden. \\ Basil Blackwell/Barnes \& Noble: 1982. \\ Pp.472. £29.50, \$35.
}

Arctic and Antarctic was a title used previously in 1939 by Colin Bertram with the subtitle The Technique of Polar Travel. In that book, published by Cambridge University Press, Bertram discussed basic principles of travel in an age when dog teams had not given way entirely to tractors and aeroplanes. Forty years later, David Sugden writes with an extensive but different experience of an Arctic and Antarctic that are no longer so remote. Commerce, armed services, scientists and governments have become increasingly active in these "resource frontier regions" of geographers.

The new volume is sub-titled $A$ Modern Geographical Synthesis. It is written to introduce undergraduates reading geography to the polar regions and to use this knowledge to test geographical concepts. The coverage is wide rather than deep in both time and space, as recognized by the author who has aimed at geographical completeness.

Sugden begins by placing the natural systems of the Arctic and Antarctic in context, with chapters on plate tectonics and climate, which are followed by regional descriptions of the glacial system, the periglacial system and the polar marine system. Photographs and diagrams as well as his own wide experience help the author to cover this broad field in a mere 140 pages. Then follows a general survey of present knowledge of environmental change in polar regions.

The second section, dealing with human systems, starts with a concise but perceptive outline of the exploration, settlement and development of polar regions. After an account of the indigenous population of the Arctic, 35 pages or so are devoted to a survey of each of five regions: Greenland and Svalbard, Arctic Canada, Alaska, the Soviet Union and Antarctica. Each starts with around four pages on "Physical constraints and resources" followed by a few pages on "The human spatial pattern", "The evolution of the spatial system", then some discussion of "The functioning of the spatial system" such as transport networks. Special topics such as comparison of Antarctic with Arctic developments follow before "Conclusions" about each region.

The geographical systems approach provides a useful link between the pattern of development in the Arctic and those in other less developed regions such as Africa. However, in Antarctica, this seems somewhat forced as scientific knowledge is presented as yet another resource. The stimulus from science in opening up the Antarctic continent is in a different class to the economic factors operating in other polar regions, and one regrets this was not given more emphasis.

The book is well written and illustrated and follows a logical pattern. This makes it useful for those not familiar with the different regions discussed. Inevitably, however, some detailed points of the author's presentation could be brought up to date or improved. For example, the large proportion of frazil ice found in many

drifting ice floes is not mentioned. Elsewhere Sugden says that the Weddell Sea drift was discovered by Ernest Shackleton when HMS Endurance (sic) was frozen into the Weddell Sea in 1915 although this had been experienced by Filchner in the Deutschland in 1911-1912 as Shackleton will have known before setting out on S.Y. Endurance. These are however blemishes of detail on a broad survey that benefits, at least in the physical sciences, from drawing on contrasts between conditions in the Arctic and Antarctic.

G. de O. Robin was formerly Director of the Scott Polar Research Institute, Cambridge.

\section{William Fowler and the elements}

\section{G. Edmunds}

Essays in Nuclear Astrophysics.

Edited by C.A. Barnes, D.D. Clayton and D.N. Schramm.

Cambridge University Press: 1982. Pp.560. Hbk £40, \$75; pbk \$29.95.

ORIGINS have their own peculiar fascination, and the origin of a particularly basic part of us - the chemical elements is no exception. The realization that nuclear fusion was the only source of energy sufficient to power the stars lea inevitably to the idea that further reactions could build up complex atomic nuclei from hydrogen and helium.

It is now over 25 years since the immensely fruitful collaboration between nuclear physics and astronomy was made apparent by the publication in 1957 of the classic systematizations of the major element production processes by Burbidge, Burbidge, Fowler and Hoyle, and by Cameron. The details of the processes have subsequently been elaborated and changed, but the broad outline remains remarkably the same. Professor Willy Fowler of the California Institute of Technology has been a prime force behind studies of nuclear astrophysics since the late 1940s. This wide-ranging collection of essays by his present and former colleagues and students is a testament to his influence and enthusiasm, particularly since almost every topic covered has been worked on or encouraged by him.

There are presently believed to be three major sites for the synthesis of the elements - the early Universe, where extreme temperatures and densities produce helium and a few light elements; the interstellar medium, where cosmic ray particles interact with interstellar atoms to produce a few more light elements; and nuclear reactions in stars, where elements including and heavier than carbon are produced, particularly during violent explosive evolution. One of the principal problems in nuclear astrophysics is the determination of the rates of reactions which occur during the normal quiescent life of a star, because the energies at which the reactions occur are such that laboratory simulations of the same conditions would give unmeasurably slow rates. Several of these essays address the problem of extrapolating laboratory measurements to the relevant energies, important for astrophysicists in predicting the composition of a star after its quiescent life, and a complicated problem in nuclear physics in its own right. The major skeleton in the stellar evolution closet has an excellent airing in an anecdotal article on the solar neutrino problem. The very low (compared to theoretical estimates) flux of observed neutrinos from one of the side chains in the solar core nuclear reactions still persists, although the impending construction of a new experiment based on a gallium detector to look at neutrinos from the main proton-proton reaction should resolve whether the astrophysicists have made their stellar models wrong or whether neutrinos do not have the properties that are usually assumed. Either way, astrophysics or particle physics will benefit from the result.

The nuclear physics of exploding stars is in some ways easier, since the reaction energies come into the range accessible in laboratory equipment. But the extreme physical conditions and hydrodynamics make the theoretical models of stars much more uncertain. It seems likely, as discussed in several of these essays, that the generation of a shock wave in the final stages of evolution of massive stars must provide sudden high temperatures for nucleosynthetic reactions and also give sufficient push to expel some of the products from the collapsing star. The details of this complex process remain uncertain, as they do for the other main types of explosion involving lower-mass, possibly binary, stars.

In recent years much excitement has come as a result of beautiful analytic chemistry which has discovered marked anomalies in relative isotope abundances in certain meteorites. Since this material is 